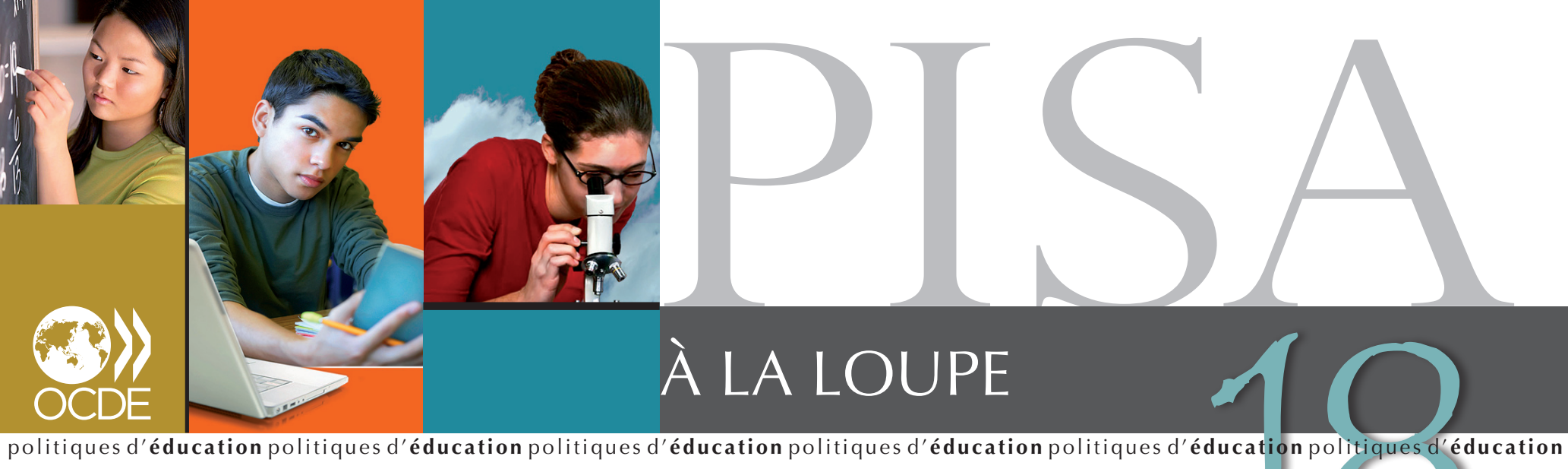

politiques d'éducation politiques d'éducation politiques d'éducation politiques d'éducation politiques d'éducation politiques d'éducation

\title{
L'offre d'activités en marge du programme de cours renforce-t-elle la motivation des élèves?
}

- Environ $90 \%$ des élèves des pays de l'OCDE fréquentent des établissements qui organisent des excursions et des activités de terrain donnant la possibilité aux élèves d'approfondir leur connaissance des principes et concepts scientifiques.

- Dans la plupart des pays, il existe une relation entre les activités en rapport avec les sciences proposées par les établissements d'enseignement en marge du programme de cours et l'amélioration de la performance des élèves, de leur perception de leurs capacités personnelles en sciences et du plaisir qu'ils retirent de l'apprentissage des sciences.

Projet de sciences. Ce nom évoque à lui seul séances de travaux pratiques et travail de groupe. La motivation et la performance des élèves dans l'apprentissage des sciences sont-elles améliorées lorsque leurs établissements d'enseignement les encouragent à participer à des projets de sciences, des expositions scientifiques, des clubs de sciences ou des excursions et des activités de terrain en rapport avec les sciences - en plus de I'enseignement du programme obligatoire de sciences ? Pour le savoir, l'enquête PISA 2006 a demandé aux chefs d'établissement quels types d'activités en rapport avec les sciences ils proposent à leurs élèves en marge du programme de cours, et a analysé la relation entre leurs réponses et la performance des élèves à l'évaluation PISA de la culture scientifique.

L'offre et le type d'activités proposées en marge du programme de cours varient fortement...
Dans les pays de l'OCDE, 89 \% des élèves fréquentent des établissements d'enseignement qui, aux dires de leurs chefs d'établissement, proposent régulièrement des excursions et des activités de terrain. En Australie, en Estonie, en Hongrie, en Italie, en Pologne, en République slovaque, en République tchèque et en Slovénie et, dans les pays partenaires, en Fédération de Russie, en Lettonie, en Lituanie, au Qatar, en Roumanie et en Thaïlande, plus de $96 \%$ des élèves fréquentent ce type d'établissements ; au Japon, en revanche, seuls $30 \%$ des élèves sont dans ce cas. En Pologne, tous les élèves fréquentent des établissements qui organisent des concours de sciences, selon les déclarations de leurs chefs d'établissement; plus de $97 \%$ des élèves sont également dans ce cas en Australie et, dans les pays partenaires, en Fédération de Russie et au Kirghizistan. En moyenne, dans les pays de l'OCDE, 56 \% des élèves fréquentent des établissements d'enseignement qui organisent des concours de sciences. Ce genre de concours n'est pas aussi répandu au Japon, où seuls $6 \%$ des élèves fréquentent des établissements qui en organisent, au Danemark (10\% des élèves) et en Norvège (16 \% des élèves). 


\begin{tabular}{|c|c|c|c|c|c|c|}
\hline & \multicolumn{5}{|c|}{$\begin{array}{l}\text { Pourcentage d'élèves scolarisés dans } \\
\text { un établissement d'enseignement } \\
\text { qui promeut I'apprentissage des sciences } \\
\text { en proposant: }\end{array}$} \\
\hline & & 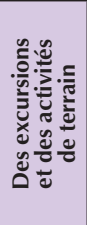 & 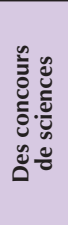 & 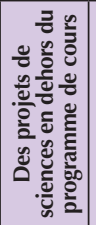 & 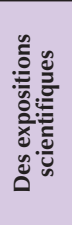 & 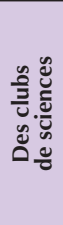 \\
\hline & & \multicolumn{5}{|c|}{$\%$} \\
\hline \multirow{34}{*}{ 岂 } & Australie & 97 & 98 & 70 & 31 & 31 \\
\hline & Autriche & 91 & 35 & 30 & 27 & 27 \\
\hline & Belgique & 91 & 52 & 48 & 35 & 5 \\
\hline & Canada & 95 & 64 & 64 & 55 & 48 \\
\hline & Chili & 74 & 36 & 47 & 44 & 39 \\
\hline & République tchèque & 97 & 78 & 50 & 61 & 47 \\
\hline & Danemark & 87 & 10 & 18 & 25 & 3 \\
\hline & Estonie & 97 & 88 & 88 & 81 & 50 \\
\hline & Finlande & 94 & 37 & 23 & 9 & 9 \\
\hline & Allemagne & 95 & 43 & 34 & 29 & 47 \\
\hline & Grèce & 87 & 67 & 23 & 9 & 11 \\
\hline & Hongrie & 97 & 84 & 38 & 69 & 72 \\
\hline & Islande & 95 & 25 & 23 & 7 & 5 \\
\hline & Irlande & 93 & 54 & 53 & 64 & 21 \\
\hline & Israël & 87 & 62 & 65 & 32 & 53 \\
\hline & Italie & 96 & 34 & 75 & 16 & 39 \\
\hline & Japon & 30 & 6 & 19 & 11 & 49 \\
\hline & Corée & 80 & 86 & 44 & 49 & 87 \\
\hline & Luxembourg & 93 & 41 & 56 & 69 & 33 \\
\hline & Mexique & 75 & 72 & 54 & 39 & 21 \\
\hline & Pays-Bas & 89 & 35 & 40 & 21 & 8 \\
\hline & Nouvelle-Zélande & 94 & 91 & 57 & 72 & 32 \\
\hline & Norvège & 94 & 16 & 42 & 36 & 1 \\
\hline & Pologne & 99 & 100 & 51 & 27 & 78 \\
\hline & Portugal & 94 & 62 & 86 & 62 & 64 \\
\hline & République slovaque & 99 & 81 & 44 & 70 & 78 \\
\hline & Slovénie & 97 & 80 & 79 & 85 & 92 \\
\hline & Espagne & 95 & 37 & 36 & 57 & 69 \\
\hline & Suède & 81 & 56 & 29 & 24 & 7 \\
\hline & Suisse & 95 & 22 & 29 & 47 & 35 \\
\hline & Turquie & 78 & 54 & 48 & 29 & 39 \\
\hline & Royaume-Uni & 87 & 72 & 60 & 35 & 73 \\
\hline & États-Unis & 92 & 58 & 65 & 50 & 73 \\
\hline & Moyenne OCDE & 89 & 56 & 48 & 42 & 41 \\
\hline \multirow{22}{*}{ 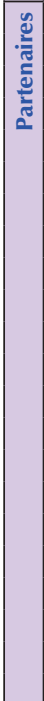 } & Argentine & 80 & 51 & 65 & 72 & 16 \\
\hline & Azerbaïdjan & 91 & 79 & 29 & 42 & 68 \\
\hline & \begin{tabular}{|l|} 
Brésil \\
\end{tabular} & 84 & 39 & 86 & 82 & 5 \\
\hline & Bulgarie & 86 & 78 & 52 & 20 & $\mathrm{a}$ \\
\hline & Taipei chinois & 89 & 72 & 71 & 73 & 76 \\
\hline & Colombie & 87 & 62 & 75 & 71 & 93 \\
\hline & Croatie & 90 & 75 & 58 & 49 & 21 \\
\hline & Hong-Kong (Chine) & 90 & 91 & 83 & 52 & 91 \\
\hline & Indonésie & 74 & 63 & 45 & 25 & 60 \\
\hline & Jordanie & 90 & 75 & 84 & 80 & 67 \\
\hline & Kirghizistan & 94 & 98 & 36 & 75 & 79 \\
\hline & Lettonie & 99 & 91 & 86 & 6 & 14 \\
\hline & Lituanie & 99 & 91 & 76 & 98 & 80 \\
\hline & Macao (Chine) & 69 & 91 & 96 & 34 & 46 \\
\hline & Monténégro & 83 & 81 & 57 & 31 & 68 \\
\hline & Qatar & 97 & 78 & 71 & 66 & 41 \\
\hline & Roumanie & 100 & 92 & 55 & 62 & 71 \\
\hline & Fédération de Russie & 99 & 98 & 80 & 83 & 84 \\
\hline & Serbie & 65 & 84 & 43 & 41 & 83 \\
\hline & Thaïlande & 96 & 93 & 89 & 97 & 84 \\
\hline & Tunisie & 78 & 49 & 51 & 56 & 83 \\
\hline & Uruguay & 83 & 32 & 60 & 57 & 33 \\
\hline
\end{tabular}

Source : Base de données PISA 2006 de l'OCDE.
Les projets de sciences en dehors du programme de cours, les expositions scientifiques et les clubs de sciences sont moins répandus dans les pays de I'OCDE : en moyenne, $48 \%$ des élèves fréquentent des établissements d'enseignement qui encouragent la participation à des projets de sciences en dehors du programme de cours ; $42 \%$, des établissements qui organisent des expositions scientifiques ;

et $41 \%$, des établissements qui ont un club de sciences.

\section{... mais il existe partout une relation entre ce type d'activités et l'amélioration de la performance des élèves.}

Dans la plupart des pays, les élèves fréquentant des établissements d'enseignement qui proposent davantage d'activités en rapport avec les sciences en marge du programme de cours ont tendance à obtenir de meilleurs résultats en sciences que les élèves fréquentant des établissements qui en proposent moins. Parmi les pays et économies dont les données sont disponibles, cette tendance s'observe dans 22 pays de l'OCDE sur 31 et dans 14 pays et économies partenaires sur 17. Les corrélations les plus fortes s'observent en Allemagne, où $15 \%$ de la variation de la performance des élèves en sciences est imputable à l'offre, par les établissements d'enseignement, d'activités en rapport avec les sciences en marge du programme de cours, et en Australie, où $13 \%$ de la variation de la performance des élèves peut s'expliquer ainsi.

Dans 21 pays de l'OCDE et dans 12 pays et économies partenaires, la relation positive entre l'offre de ce type d'activités par les établissements d'enseignement et la performance des élèves en sciences persiste même après contrôle du milieu socio-économique des élèves. En revanche, aux États-Unis, les élèves fréquentant des établissements d'enseignement qui proposent moins d'activités de ce type ont tendance à obtenir de meilleurs résultats en sciences, après contrôle du milieu socio-économique des élèves, tandis qu'au Monténégro, cette relation est négative, tant avant qu'après contrôle du milieu socio-économique des élèves. 
Même après contrôle du milieu

socio-économique des établissements et des élèves, en moyenne, dans 8 pays de l'OCDE, 4 pays partenaires et 1 économie partenaire, les élèves fréquentant des établissements d'enseignement qui proposent davantage d'activités en marge du programme de cours tendent à obtenir de meilleurs résultats que les élèves fréquentant des établissements qui proposent moins ce type d'activités. Dans nombre de pays et économies, I'avantage de performance des établissements d'enseignement qui proposent davantage d'activités en rapport avec les sciences en marge du programme de cours disparaît après contrôle du milieu socio-économique des élèves et des établissements. Cela tient au fait que les établissements d'enseignement qui proposent davantage d'activités de ce type sont, en général, également favorisés sur le plan socio-économique et, bénéficient, par conséquent, d'autres caractéristiques également liées à une meilleure performance aux évaluations PISA.

Les bénéfices sont également visibles dans les attitudes des élèves.

Les élèves fréquentant des établissements d'enseignement qui proposent davantage d'activités en rapport avec les sciences en marge du programme de cours ont non seulement tendance à obtenir de meilleurs résultats en sciences, mais également à faire preuve d'attitudes plus positives à l'égard des sciences. Ils croient en leurs capacités personnelles pour mener à bien des tâches liées aux sciences (soit la perception des capacités personnelles en sciences) et retirent du plaisir de l'apprentissage des sciences. La perception des capacités personnelles et le plaisir jouent un rôle majeur dans l'apprentissage ; en effet, il est prouvé que ces deux facteurs influent de façon significative sur la façon dont les élèves se fixent leurs objectifs et choisissent leurs stratégies d'apprentissage.

Source : Base de données PISA 2006 de l'OCDE.

Remarque : les analyses examinant la relation entre les activités en rapport avec les sciences proposées en marge du programme de cours et la performance en sciences, la perception des capacités personnelles en sciences et le plaisir apporté par les sciences sont dérivées de l'indice composite d'activités scolaires visant à promouvoir l'apprentissage des sciences.

Consulter OCDE, PISA 2006 : Les compétences en sciences, un atout pour réussir : Volume II : Données, tableau 5.18

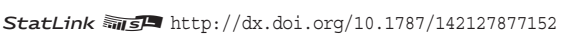

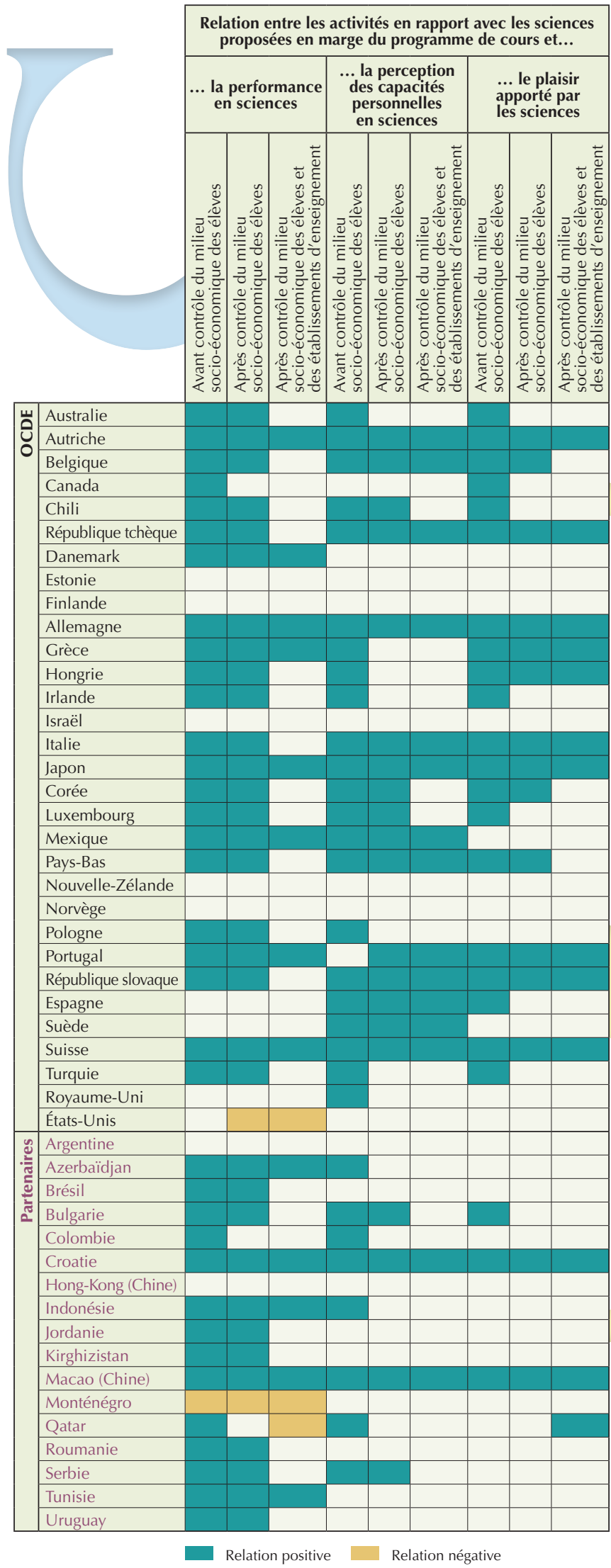


Dans 22 pays de l'OCDE, 7 pays partenaires et 1 économie partenaire, les élèves fréquentant des établissements d'enseignement proposant davantage d'activités de ce type tendent à faire état d'une plus haute opinion de leurs capacités personnelles en sciences; et dans 20 pays de l'OCDE, 2 pays partenaires et 1 économie partenaire, ils retirent également davantage de plaisir de l'apprentissage des sciences.

Après contrôle du milieu socio-économique des élèves et des établissements, la relation positive avec la perception des capacités personnelles en sciences persiste dans 13 pays de l'OCDE, 1 pays partenaire et 1 économie partenaire, et la relation positive avec le plaisir apporté par I'apprentissage des sciences perdure dans 10 pays de I'OCDE, 1 pays partenaire et 2 économies partenaires. On n'observe de relation négative entre l'offre d'activités en rapport avec les sciences en marge du programme de cours et les attitudes positives à l'égard de l'apprentissage des sciences dans aucun pays ni économie.
Pour déterminer la position des élèves sur l'indice de perception des capacités personnelles en sciences, l'enquête PISA les a interrogés sur la mesure dans laquelle ils s'estiment capables : i) « d'identifier la question scientifique qui est à la base d'un article de journal portant sur un problème de santé »; ii) « d'expliquer pourquoi les tremblements de terre sont plus fréquents dans certaines régions que dans d'autres »; iii) « de décrire le rôle des antibiotiques dans le traitement des maladies »; $i v)$ " de déterminer quelle est la question scientifique liée au traitement des déchets »; v) « de prévoir en quoi des changements apportés à l'environnement affecteront la survie de certaines espèces »; vi) " d'interpréter des informations scientifiques fournies sur l'étiquette des produits alimentaires »; vii) " de discuter sur la façon dont des données nouvelles pourraient modifier leur point de vue sur la probabilité qu'il existe de la vie sur Mars » ; et viii) « de déterminer quelle est la meilleure de deux explications sur la formation de pluies acides ». Ces items étaient assortis de quatre catégories de réponse : "j’y arriverais facilement »; "j'y arriverais avec un peu d'effort »; " cela ne me serait pas facile d'y arriver seul(e)» ; et enfin, « je n'y arriverais pas ».

Pour déterminer la position des élèves sur l'indice de plaisir apporté par les sciences, l'enquête PISA les a interrogés sur leur degré d'assentiment aux affirmations suivantes : $i$ ) « je trouve généralement agréable d'apprendre des notions de <sciences au sens large $>$ "; ii) " j'aime lire des textes qui traitent de <sciences au sens large $>$ "; iii) « cela me plaît d'avoir à résoudre des problèmes en <sciences au sens large $>$ » ; iv) « je prends plaisir à acquérir de nouvelles connaissances en <sciences au sens large > »; et $v$ ) " cela m'intéresse d'apprendre des choses en <sciences au sens large $>»$. Ces items étaient assortis de quatre catégories de réponse : « tout à fait d'accord " ; " d'accord » ; " pas d'accord »; et enfin, " pas du tout d'accord ».

Pour conclure: L'enquête PISA ne permet pas de déterminer si c'est l'offre d'activités en rapport avec les sciences en marge du programme de cours qui améliore les attitudes des élèves à l'égard de l'apprentissage des sciences ou si ce sont les élèves affichant des attitudes plus positives à l'égard des sciences qui sont attirés par les établissements d'enseignement qui proposent davantage ce type d'activités; l'une comme l'autre de ces hypothèses pourraient être valables. Pour autant, ce que PISA montre bien, c'est que ce type d'activités a un impact positif non seulement sur la performance des élèves, mais aussi sur les attitudes des élèves à l'égard de l'apprentissage et leur perception de leurs capacités personnelles.

\section{Pour tout complément d'information}

Contacter Miyako Ikeda (Miyako.Ikeda@oecd.org)

Consulter PISA 2006 : Les compétences en sciences, un atout pour réussir : Volume I : Analyse des résultats, Éditions OCDE ; PISA 2006 : Les compétences en sciences, un atout pour réussir : Volume II : Données, Éditions OCDE.

Voir

www.pisa.oecd.org

www.oecd.org/pisa/infocus
Prochain numéro

Existe-t-il vraiment une « seconde chance » en matière d'éducation? 